\title{
ATLAS Tile Calorimeter time calibration, monitoring and performance in Run 2
}

\author{
Michaela Mlynarikova*, on behalf of the ATLAS Tile Calorimeter System \\ ${ }^{*}$ Department of Physics, Northern Illinois University, DeKalb, IL, United States of America \\ E-mail: michaela.mlynarikova@cern.ch
}

The Tile Calorimeter is the central hadronic calorimeter of the ATLAS experiment at the Large Hadron Collider at CERN. This sampling device uses steel plates as absorber and scintillating tiles as active medium. Its response is calibrated to the electromagnetic energy scale by means of several dedicated calibration systems. Accurate time calibration is important for energy reconstruction and non-collision background removal. The principle, procedure, and results of time calibration using collision data are presented. Monitoring of the time calibration with a laser system and collision data are discussed as well. Finally, the time resolution as measured with jets in Run 2 is presented.

40th International Conference on High Energy physics - ICHEP2020

July 28 - August 6, 2020

Prague, Czech Republic (virtual meeting) 


\section{Introduction}

The Tile Calorimeter (TileCal) is the central hadronic calorimeter of the ATLAS experiment [1] at the Large Hadron Collider (LHC) at CERN. TileCal is a sampling calorimeter built of plastic scintillating tiles regularly spaced between steel absorber plates. Scintillation light generated by charged particles traversing a tile is collected and delivered to a separate photomultiplier tube (PMT) at each end by a wavelength-shifting (WLS) fiber. The readout cell geometry is given by a group of WLS fibers from individual tiles coupled to PMTs. Usually, a cell is read out by two PMTs, each corresponding to a single channel.

TileCal measures the energy and direction of isolated hadrons, $\tau$-leptons and jets, and provides essential input to the missing transverse energy reconstruction. The time when particles and jets hit the calorimeter cell is recorded as well. This information is essential for the removal of signals which do not originate from proton-proton $(p p)$ collisions. Such information is also needed for time-of-flight measurements of hypothetical heavy slow particles that would reach the calorimeter.

\subsection{Signal reconstruction}

The analog pulse from each PMT is shaped and split into two branches: high- and low-gain amplifier, with a gain ratio of 64:1. Each pulse is then sampled and digitized every $25 \mathrm{~ns}$ and the amplitude $A$, which is proportional to the deposited energy, and time $t_{0}$ are reconstructed. An Optimal Filter (OF) method is used for physics data reconstruction [2]:

$$
A=\sum_{i=1}^{7} a_{i} S_{i}, \quad t_{0}=\frac{1}{A} \sum_{i=1}^{7} b_{i} S_{i},
$$

where $S_{i}$ represent individual values of the sampled pulse. The OF constants $a_{i}, b_{i}$, were derived from a reference pulse shape, and $t_{0}$ is a measurement of the pulse arrival phase and determines the time calibration.

\section{Time calibration, monitoring and performance}

The time calibration aims to set the phase in each channel so that a particle traveling from the ATLAS interaction point at the speed of light produces a signal with a measured time equal to zero. Jets originating from $p p$ collisions are used for the final time calibration. To avoid possible bias from non-collision background, only cells/channels used in the reconstruction of these jets are considered. The average cell time depends slightly on the energy deposited in a cell [3]. Therefore, a requirement on channel energy, $2<E_{\text {channel }}<4 \mathrm{GeV}$, was chosen as a trade-off between sufficient data statistics and Gaussian shape of the time response. The time constants are determined as a Gaussian mean of the time distribution in each channel.

During physics data taking, laser calibration events [4] and events containing jets are used to monitor the channel time stability. Laser pulses are shot in the LHC empty bunch-crossings and a dedicated tool set up to spot sudden changes in time synchronization is used to analyze the output. A complementary time monitoring tool that utilizes $p p \rightarrow$ jets data is used to cross check the laser results. 

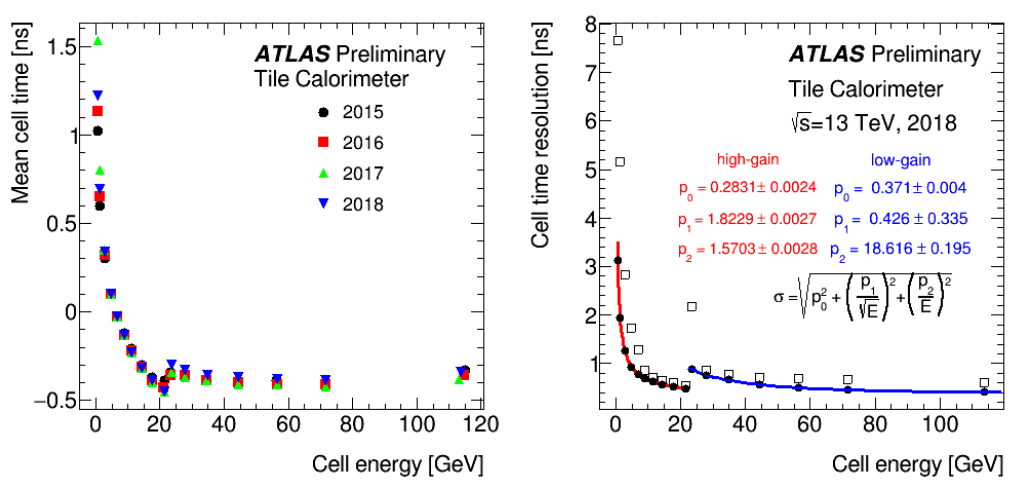

Figure 1: Left: The mean cell time as measured with jet events as a function of energy deposited in the cell [5]. Right: Cell time resolution measured with jets [5]. Full circles indicate Gaussian widths and open squares indicate the RMS of the underlying time spectra. The discontinuity close to $22 \mathrm{GeV}$ corresponds to the transition between high and low gain amplitude readout.

These results are available shortly after the end of each physics run. Therefore, time constant corrections can be applied before the collision data are re-processed and used in physics analyses.

As shown in the left plot of Figure 1, the mean cell time measured with jets depends on the energy deposited in the cells. Results from each year demonstrate a stable performance of the time calibration during the LHC Run 2 data taking. Results of time resolution studies using jet events in the 2018 data are shown in the right plot of Figure 1. The RMS values are systematically higher than Gaussian widths, indicating non-Gaussian tails in the measured time distribution that originate from the concurrent interactions in neighboring bunch-crossings.

\section{Conclusions}

Methods used for the time calibration and time stability monitoring of the ATLAS Tile Calorimeter during the LHC Run 2 are summarized and the main performance results are highlighted. The mean cell time and time resolution as functions of the deposited energy in jet data showed a stable performance over the Run 2 data taking period.

\section{References}

[1] ATLAS Collaboration, The ATLAS Experiment at the CERN Large Hadron Collider, JINST 3 (2008), S08003.

[2] A. Valero, Implementation and performance of the signal reconstruction in the ATLAS Hadronic Tile Calorimeter, Phys. Procedia 37 (2012), 1765-1771.

[3] ATLAS Collaboration, Operation and performance of the ATLAS Tile Calorimeter in Run 1, Eur. Phys. J. C 78 (2018) no.12, 987, [arXiv:1806.02129].

[4] ATLAS Tile Calorimeter System, The Laser calibration of the Atlas Tile Calorimeter during the LHC run 1, JINST 11 (2016) no.10, T10005, [arXiv:1608.02791].

[5] Tile Calorimeter public plots, https://twiki.cern.ch/twiki/bin/view/AtlasPublic/TileCaloPublicResults. 